\title{
Approximation methods for lognormal characteristic functions
}

DOI:

10.1080/00949655.2018.1530775

\section{Document Version}

Accepted author manuscript

Link to publication record in Manchester Research Explorer

\section{Citation for published version (APA):}

Wiegand, M., \& Nadarajah, S. (2018). Approximation methods for lognormal characteristic functions. Journal of Statistical Computation and Simulation, 88(18), 3650-3663. https://doi.org/10.1080/00949655.2018.1530775

\section{Published in:}

Journal of Statistical Computation and Simulation

\section{Citing this paper}

Please note that where the full-text provided on Manchester Research Explorer is the Author Accepted Manuscript or Proof version this may differ from the final Published version. If citing, it is advised that you check and use the publisher's definitive version.

\section{General rights}

Copyright and moral rights for the publications made accessible in the Research Explorer are retained by the authors and/or other copyright owners and it is a condition of accessing publications that users recognise and abide by the legal requirements associated with these rights.

\section{Takedown policy}

If you believe that this document breaches copyright please refer to the University of Manchester's Takedown Procedures [http://man.ac.uk/04Y6Bo] or contact uml.scholarlycommunications@manchester.ac.uk providing relevant details, so we can investigate your claim.

\section{OPEN ACCESS}




\title{
Approximation methods for lognormal characteristic functions
}

\author{
Martin Wiegand and Saralees Nadarajah \\ School of Mathematics, University of Manchester, Manchester M13 9PL, UK
}

November 22, 2018

\begin{abstract}
The characteristic function of the lognormal distribution is of interest in a number of scientific fields yet an analytic solution remains elusive, making reliable and efficient approximations necessary. In this article, we build on the results of N. C. Beaulieu and A. Saberali in "New approximations to the lognormal characteristic function", by introducing a Taylor- and Bessel function-based partial expansion of the integrand and a Chebyshev quadrature approach. Through computer simulations we show that the Taylor expansion remains accurate and efficient for all commonly computed values, and specify the range of values for which the other two approaches show a significantly stronger performance.
\end{abstract}

\section{Introduction}

The lognormal distribution features in a number of application fields in science, such as biology, medicine, communications and finance [14]. For example, its distribution along with other heavy-tailed distributions is used to model the severity of a loss incident in operational risk management, or the income distribution among populations [10].

The fact that the logarithm of a product of random variables is the sum of its logarithmic factors, makes the lognormal distribution one of the most commonly encountered distributions. Since the sum of random variables asymptotically exhibits normal distribution, the product of said random variables itself will have a lognormal distribution as a direct result of the Central Limit Theorem (see also Asmussen et al. [2]).

Let $Z$ be a random variable of lognormal distribution, with $z>0,-\infty<\mu<\infty$ and $\sigma>0$. Then the probability density function is defined in ??:

$f_{Z}(z)=\frac{1}{\sqrt{2 \pi} \sigma z} \exp \left[\frac{(\log (z)-\mu)^{2}}{2 \sigma^{2}}\right]$.

We write $Z \sim L N\left(\mu, \sigma^{2}\right)$, with $\mu$ and $\sigma^{2}$ being the mean and variance, respectively.

The importance of the characteristic function $(\mathrm{CF})$ rests upon its ability to encapsulate the behavior of the probability density function of its underlying random variable. The CF of $Z$ is commonly stated as

$\Phi_{Z}(\omega)=\int_{0}^{\infty} \frac{1}{\sqrt{2 \pi} \sigma z} \exp \left[-\frac{(\log (z)-\mu)^{2}}{2 \sigma^{2}}\right] e^{i \omega z} d z$,

where $i=\sqrt{-1}$. Currently, no closed form of this integral is known, creating the necessity for accurate and fast approximations. Depending on the variance, the integrand can be a highly oscillatory function with a heavy tail. This may make the computation by standard numerical approaches time consuming and unstable.

A number of series representations have been put forth over time, often originating out of the field of communications. Especially interesting for wireless communications, the distribution has been employed to model large-scale signal fading, the interference of mobile and other wireless networks (Tellambura and Senaratne [17]) or shadowing effects in radio transmission (Beaulieu [6]). Earlier approximations and their applications include Barakat's approach which aimed to model optical propagation through the atmosphere [3. Depending on their intended applications, these approximations focus on a special range of parameter values. We will investigate the performance of the newly proposed approaches accordingly. In communications the ratio of power is expressed in decibels $(\mathrm{dB})$, the range of interest is therefore $\sigma_{y}=10 / \log 10 \sigma$ [12] from $10^{-2} \mathrm{~dB}$ to $13 \mathrm{~dB}(0.0023<\sigma<3$ as seen in [18], [7] $)$ in which the methods are required to operate reliably.

This paper is structured into four parts. In Section 2, we begin by introducing a partial Taylor expansion into the integrand and determine its analytic solution for finite intervals of appropriate length. Secondly, we propose another approach based on the series expansion of the exponential function utilizing modified Bessel functions. We follow this 
up with a simulation study of different techniques, based on both the central limit theorem and series expansions. We will compare computational efficiency as well as absolute and relative error measures.

In Section 3, we cover quadrature methods, such as the Chebyshev type quadrature, which we introduce to an integral formulation of the CF, as proposed by Gubner [11. Once again we run simulations to compare efficiency and stability of the different approaches to one another. We close with a terse statement about the performances and suitable parameter regions of all investigated methods.

\section{Expansion approaches for the CF}

\subsection{Partial Taylor expansions on finite intervals}

We reformulate by splitting up the CF into real and imaginary parts for convenient handling. We will only examine the computation of the real part for now, as the imaginary part can be evaluated analogously, by substituting $z$ for $x=w z$, so that the second factor function is not dependent on any other variables. As previously stated throughout literature [8], we may assume $\mu=0$ without loss of generality.

Note that the following holds:

$$
\begin{aligned}
\Phi_{Z}(\omega) & =\int_{0}^{\infty} \frac{1}{\sqrt{2 \pi} \sigma z} \exp \left[-\frac{\log \left(\frac{z}{\omega}\right)^{2}}{2 \sigma^{2}}\right][\cos (\omega z)+i \sin (\omega z)] d z \\
& =\frac{1}{\sqrt{2 \pi} \sigma} \int_{0}^{\infty} \frac{1}{z} \exp \left[-\frac{(\log (x)-\log (w))^{2}}{2 \sigma^{2}}\right] \cos (x) d x+i \operatorname{Im}\left(\Phi_{Z}(\omega)\right) \\
& =\frac{1}{\sqrt{2 \pi} \sigma} \int_{0}^{\infty} \frac{1}{z} \exp \left[-\frac{\left(\log (x)^{2}-2 \log (w) \log (x)+\log (w)\right)^{2}}{2 \sigma^{2}}\right] \cos (x) d x+i \operatorname{Im}\left(\Phi_{Z}(\omega)\right) \\
& =\frac{\exp \left[-\frac{\log (\omega)}{2 \sigma^{2}}\right]}{\sqrt{2 \pi} \sigma} \int_{0}^{\infty} \frac{1}{z} \exp \left[-\frac{\log (x)^{2}}{2 \sigma^{2}}\right] \exp \left[\frac{1}{\sigma^{2}} \log (\omega) \log (x)\right] \cos (x) d x+i \operatorname{Im}\left(\Phi_{Z}(\omega)\right) \\
& =\underbrace{\frac{\exp \left[-\frac{\log (\omega)}{2 \sigma^{2}}\right]}{\sqrt{2 \pi} \sigma}}_{c_{(\omega, \sigma)}} \int_{0}^{\infty} \exp \left[-\frac{\log (x)^{2}}{2 \sigma^{2}}\right] x^{\frac{1}{\sigma^{2}} \log (\omega)-1} \cos (x) d x+i \operatorname{Im}\left(\Phi_{Z}(\omega)\right)
\end{aligned}
$$

The integral above still does not have an analytic solution, which means that from this point on we will work on approximate representations of the $\mathrm{CF}$, rather than exact formulations. We split the domain of integration into an infinite number of sections $\left[a_{k}, a_{k+1}\right]$ with $k=0, \ldots, \infty$ and $a_{k}=k t$ where $t$ is the interval width:

$\Phi_{Z}(\omega)=c_{(\omega, \sigma)} \sum_{k=0}^{\infty} \int_{a_{k}}^{a_{k+1}} \exp \left[-\frac{\log (x)^{2}}{2 \sigma^{2}}\right] x^{\frac{1}{\sigma^{2}} \log (\omega)-1} \cos (x) d x$.

In each interval, we now introduce a straightforward Taylor series representation of the cosine function (note that $\left.\cos (x)^{(j)}=\cos \left(x+j \frac{\pi}{2}\right)\right)$. We do this in order to gain sum terms for which the integral has a closed form solution. Given the shape of the integrand, which is oscillating around zero with a diminishing amplitude as $x$ grows larger, the interval size of each individual integral section needs to be chosen appropriately with respect to the stability of the section's integration (the larger the intervals, the harder it is to find an accurate, stable approximation).

There has to be a balance between interval size and the order of the Taylor expansion. The series expansion is centered around the midpoint of the corresponding interval $\bar{a}_{k}=\frac{a_{k+1}-a_{k}}{2}$. Hence, we obtain the following series representation:

$\Phi_{Z}(\omega)=c_{(\omega, \sigma)} \sum_{k=0}^{\infty} \int_{a_{k}}^{a_{k+1}} \exp \left[-\frac{\log (x)^{2}}{2 \sigma^{2}}\right] x^{\frac{1}{\sigma^{2}} \log (\omega)-1} \sum_{j=0}^{\infty} \frac{\cos \left(\bar{a}_{k}+j \frac{\pi}{2}\right)}{j !}\left(x-\bar{a}_{k}\right)^{j} d x$.

Since the exponential term will eventually outweigh the polynomials or the cosine function and cause the integrand to be zero, we can take a sufficiently large finite number of intervals $m$ as an approximation. We may also interrupt the Taylor series after $n$ steps, leaving us with a finite double sum. We then rearrange the terms as follows:

$\Phi_{Z}(\omega) \approx c_{(\omega, \sigma)} \sum_{k=0}^{m} \int_{a_{k}}^{a_{k+1}} \exp \left[-\frac{\log (x)^{2}}{2 \sigma^{2}}\right] x^{\frac{1}{\sigma^{2}} \log (\omega)-1} \sum_{j=0}^{n} \frac{\cos \left(\bar{a}_{k}+j \frac{\pi}{2}\right)}{j !}\left(x-\bar{a}_{k}\right)^{j} d x$ 


$$
\begin{aligned}
& =c_{(\omega, \sigma)} \sum_{k=0}^{m} \sum_{j=0}^{n} \frac{\cos \left(\bar{a}_{k}+j \frac{\pi}{2}\right)}{j !} \int_{a_{k}}^{a_{k+1}} \exp \left[-\frac{\log (x)^{2}}{2 \sigma^{2}}\right] x^{\frac{1}{\sigma^{2}} \log (\omega)-1}\left(x-\bar{a}_{k}\right)^{j} d x \\
& =c_{(\omega, \sigma)} \sum_{k=0}^{m} \sum_{j=0}^{n} \frac{\cos \left(\bar{a}_{k}+j \frac{\pi}{2}\right)}{j !} \int_{a_{k}}^{a_{k+1}} \exp \left[-\frac{\log (x)^{2}}{2 \sigma^{2}}\right] x^{\frac{1}{\sigma^{2}} \log (\omega)-1} \sum_{h=0}^{j}\left(\begin{array}{l}
j \\
h
\end{array}\right) x^{h}\left(-\bar{a}_{k}\right)^{j-h} d x \\
& =c_{(\omega, \sigma)} \sum_{k=0}^{m} \sum_{j=0}^{n} \sum_{h=0}^{j} \frac{\cos \left(\bar{a}_{k}+j \frac{\pi}{2}\right)}{j !}\left(\begin{array}{l}
j \\
h
\end{array}\right)\left(-\bar{a}_{k}\right)^{j-h} \int_{a_{k}}^{a_{k+1}} \exp \left[-\frac{\log (x)^{2}}{2 \sigma^{2}}\right] x^{\frac{1}{\sigma^{2}} \log (\omega)-1+h} d x .
\end{aligned}
$$

The transformations in ?? are essentially a rearrangement of the sum terms. We expand the monomials of the Taylor expansion by means of the binomial theorem. We are left with a simplified integrand, for which we can find a closed form solution. The remaining composite function of exponential and polynomial factors can hence be expressed as follows:

$\int \exp \left[-\beta \log (x)^{2}\right] x^{\alpha} d x=-\frac{\sqrt{\pi}}{2 \sqrt{\beta}} e^{\frac{(\alpha+1)^{2}}{4 \beta}} \operatorname{Erf}\left[\frac{\alpha-2 \beta \log (x)+1}{2 \sqrt{\beta}}\right]$,

where $\alpha=\frac{\log (\omega)}{\sigma^{2}}-1+h, \beta=\frac{1}{2 \sigma^{2}}$ and $\operatorname{Erf}(\cdot)$ denotes the error function. We therefore conclude:

$$
\begin{aligned}
I(\omega, \sigma, h, k) & :=\int_{a_{k}}^{a_{k+1}} \exp \left[-\frac{\log (x)^{2}}{2 \sigma^{2}}\right] x^{\frac{1}{\sigma^{2}} \log (\omega)-1+h} d x \\
& =\exp \left[\frac{h^{2} \sigma^{2}+\log (\omega)^{2}}{2 \sigma^{2}}\right] \sqrt{\frac{\pi}{2}} \sigma w^{h}\left[\operatorname{Erf}\left(\frac{h \sigma^{2}-\log a_{k}+\log \omega}{\sqrt{2} \sigma}\right)-\operatorname{Erf}\left(\frac{h \sigma^{2}-\log a_{k+1}+\log \omega}{\sqrt{2} \sigma}\right)\right] .
\end{aligned}
$$

Expression ?? can then be efficiently evaluated using standard numerical methods in Mathematica or similar software.

Hence, we have an approximation dependent on the three parameters $m, n$ and $t$, being the number of intervals, order of Taylor expansion and interval width, respectively:

$\Phi_{Z}(\omega) \approx \widehat{\Phi}_{Z}(\omega)=c_{(\omega, \sigma)} \sum_{k=0}^{m} \sum_{j=0}^{n} \sum_{h=0}^{j} \frac{\cos \left(\bar{a}_{k}+j \frac{\pi}{2}\right)}{j !}\left(\begin{array}{l}j \\ h\end{array}\right)\left(-\bar{a}_{k}\right)^{j-h} I(\omega, \sigma, h, k)$.

To compute the imaginary part of the CF the cosine function in ?? may simply be replaced by a sine function.

\subsection{Bessel function series expansion}

We begin with expression ??, and introduce a series expansion for the exponential function in ??:

$e^{-z}=I_{0}(z)+2 \sum_{k=1}^{\infty} I_{k}(z)$

where $I_{k}(z)$ denotes the modified I-Bessel function.

The series representation can easily be altered to replace the trigonometric functions as well, the result of which can be seen in ??:

$\cos (z)=J_{0}(z)+2 \sum_{k=1}^{\infty}(-1)^{k} I_{2 k}(z)$

where $J_{k}(z)$ denotes the J-Bessel function.

We substitute expression ?? into definition ?? and obtain a new series representation ??:

$$
\begin{aligned}
\Phi_{Z}^{r e}(\omega) & =c_{(\omega, \sigma)} \int_{0}^{\infty} \exp \left[-\frac{\log (x)^{2}}{2 \sigma^{2}}\right] x^{\frac{1}{\sigma^{2}} \log (\omega)-1}\left[J_{0}(z)+2 \sum_{k=1}^{\infty}(-1)^{k} I_{2 k}(z)\right] d z \\
& =c_{(\omega, \sigma)}\left\{\int_{0}^{\infty} \exp \left[-\frac{\log (x)^{2}}{2 \sigma^{2}}\right] x^{\frac{1}{\sigma^{2}} \log (\omega)-1} J_{0}(z) d z+2 \int_{0}^{\infty} \exp \left[-\frac{\log (x)^{2}}{2 \sigma^{2}}\right] x^{\frac{1}{\sigma^{2}} \log (\omega)-1} \sum_{k=1}^{\infty}(-1)^{k} I_{2 k}(z) d z\right\} \\
& =c_{(\omega, \sigma)}\left\{\int_{0}^{\infty} \exp \left[-\frac{\log (x)^{2}}{2 \sigma^{2}}\right] x^{\frac{1}{\sigma^{2}} \log (\omega)-1} \sum_{m=0}^{\infty} \frac{(-1)^{m}}{(m !)^{2}}\left(\frac{z}{2}\right)^{2 m} d z\right.
\end{aligned}
$$




$$
\begin{aligned}
& \left.+2 \int_{0}^{\infty} \exp \left[-\frac{\log (x)^{2}}{2 \sigma^{2}}\right] x^{\frac{1}{\sigma^{2}} \log (\omega)-1} \sum_{k=1}^{\infty}(-1)^{k} \sum_{m=0}^{\infty} \frac{(-1)^{m}}{m !(m+2 k) !}\left(\frac{z}{2}\right)^{2 m+2 k} d z\right\} \\
\approx c_{(\omega, \sigma)} & \left\{\sum_{m=0}^{r} \frac{(-1)^{m}}{(m !)^{2}}\left(\frac{1}{2}\right)^{2 m} \int_{0}^{c} \exp \left[-\frac{\log (x)^{2}}{2 \sigma^{2}}\right] x^{\frac{1}{\sigma^{2}} \log (\omega)-1+2 m} d z\right. \\
& \left.+2 \sum_{k=1}^{b} \sum_{m=0}^{r} \frac{(-1)^{(m+k)}}{m !(m+2 k) !}\left(\frac{1}{2}\right)^{2 m+2 k} \int_{0}^{c} \exp \left[-\frac{\log (x)^{2}}{2 \sigma^{2}}\right] x^{\frac{1}{\sigma^{2}} \log (\omega)-1+2(m+k)} d z\right\} .
\end{aligned}
$$

By discontinuing the series representation after a finite amount of sum terms we are once again evaluating an approximation instead of an exact expansion. The cosine expansion as well as the Bessel function expansion are convergent, therefore the number of sum terms can be chosen to balance out accuracy and computation speed, in accordance with the required approximation properties. The parameters $b, r, c$ denote the number of cosine series terms, exponential expansion terms (containing the J-Bessel function) and the upper integration limit, respectively. We have empirically found the appropriate number to be between 15 and 30 sum terms per interval.

The Bessel expansion has proven to be stable enough for the integration interval not to be segmented. However, to ensure the desired upper integration limit $c$ can be reached, a sufficiently large amount of series terms are evaluated. If $c$ is chosen too large (or $b, r$ too small) we may be integrating a strongly deviating approximate integrand.

The integral part which requires numerical evaluation is expressed in ??:

$\operatorname{Int}(h, \sigma, c):=\int_{0}^{c} e^{\frac{-\log (z)^{2}}{2 \sigma^{2}}} x^{h} d z=\exp \left[(h+1)^{2} \frac{\sigma^{2}}{2}\right] \sigma \sqrt{\frac{\pi}{2}} \operatorname{Erfc}\left(\frac{(1+h) \sigma^{2}-\log (c)}{\sqrt{2} \sigma}\right)$,

where $\operatorname{Erfc}(\cdot)$ denotes the complementary error function. Hence, the new approximation follows in equation ??:

$$
\begin{aligned}
\widehat{\Phi}_{Z}^{r e}(\omega)=c_{(\omega, \sigma)}\left[\sum_{m=0}^{r}\right. & \frac{(-1)^{m}}{(m !)^{2}}\left(\frac{1}{2}\right)^{2 m} \operatorname{Int}\left(\frac{1}{\sigma^{2}} \log (\omega)-1+2 m, \sigma, c\right) \\
& \left.+2 \sum_{k=1}^{b} \sum_{m=0}^{r} \frac{(-1)^{(m+k)}}{m !(m+2 k) !}\left(\frac{1}{2}\right)^{2 m+2 k} \operatorname{Int}\left(\frac{1}{\sigma^{2}} \log (\omega)-1+2(m+k), \sigma, c\right)\right] .
\end{aligned}
$$

This approximation relies on a double instead of a triple sum, thus requiring significantly fewer evaluations of the computationally expensive error function.

\subsection{Numerical results for expansion approaches}

We may numerically generate accurate results for arbitrary values of $\omega$ to assess the quality of our approximations by taking the mean of a randomly generated sample of lognormal random variables $Z \sim L N\left(0, \sigma^{2}\right)$ as in Equation ??:

$\operatorname{LNSim}_{Z, n}(\omega)=\frac{1}{n} \sum_{k=1}^{n} e^{i \omega Z_{k}}$.

We have determined that a sample size of $n=10^{7}$ is sufficient for our needs, as larger sample sizes have not influenced the outcome of the simulation. The $\sigma$ values were selected according to [8] and [11], where a wide range of commonly used parameter values were investigated.

We compare the different methods to one another under the criteria of accuracy, computational speed and stability. To be more precise, we compare the aggregated absolute and relative errors for $\omega=1,2, \ldots, p$ of both the imaginary and real parts, as well as the CPU time using the Timing function in Mathematica. We denote the number of equidistantly spaced evaluation points by the parameter $p$. We have listed the error measures for different values of the $\omega$ parameter, so we can get a better idea of how the approximation behavior differs for smaller or larger values. We have omitted the individual run times for $p=10$ and $p=20$, since the computational effort for more evaluation points adds up linearly. Thus the run times for $p=10$ for example are one third of the overall run times. Tables ?? through ?? list the outcomes of simulations for different standard deviations. 


\begin{tabular}{rl|c|cc|cc} 
& & & \multicolumn{2}{c}{ Real part } & \multicolumn{2}{c}{ Imaginary part } \\
& & CPU time & Absolute error & Relative error & Absolute error & Relative error \\
\hline & ABT & 0.312002 & nc & nc & nc & nc \\
$\mathbf{p = 3 0}$ & AHG & 0.218401 & 0.404562 & 6.970940 & 0.317532 & 3.325102 \\
& ATE & 0.202801 & 0.098071 & 1.533669 & 0.094710 & 0.906951 \\
& ABE & 1.248008 & 0.043085 & 0.907838 & 0.081884 & 0.743698 \\
\hline & ABT & - & 3.557634 & 71.452960 & 3.989855 & 39.566053 \\
$\mathbf{p}=\mathbf{2 0}$ & AHG & - & 0.315587 & 4.104002 & 0.164405 & 1.339807 \\
& ATE & - & 0.081599 & 1.021646 & 0.066494 & 0.548009 \\
& ABE & - & 0.026484 & 0.355239 & 0.057447 & 0.426060 \\
\hline & ABT & - & 0.524513 & 3.560700 & 0.586144 & 3.484317 \\
& AHG & - & 0.152516 & 1.217180 & 0.059637 & 0.320366 \\
& ATE & - & 0.040261 & 0.279177 & 0.024702 & 0.150795 \\
& ABE & - & 0.011364 & 0.084232 & 0.030353 & 0.171276
\end{tabular}

Table 1: Standard deviation $\sigma=2.30$, error in comparison to simulation of size $n=10^{7}$. ALS left out.

The abbreviations read as follows: ATE and ABE are the partial Taylor and Bessel expansions, respectively, ALS denotes the localised expansion formula and AHG denotes the hypergeometric function approximation, both put forth by Beaulieu and Saberali [8]. ABT stands for the truncated lognormal function as introduced by Beaulieu [6].

\begin{tabular}{cc|c|cc|cc} 
& & & \multicolumn{2}{|c}{ Real part } & \multicolumn{2}{c}{ Imaginary part } \\
& & CPU time & Absolute error & Relative error & Absolute error & Relative error \\
\hline & ALS & $\sim 0$ & nc & $\mathrm{nc}$ & $\mathrm{nc}$ & $\mathrm{nc}$ \\
& ABT & 0.280802 & $\mathrm{nc}$ & $\mathrm{nc}$ & $\mathrm{nc}$ & $\mathrm{nc}$ \\
$\mathbf{p = 3 0}$ & AHG & 0.405603 & 0.148581 & 9.666702 & 0.121496 & 2.135366 \\
& ATE & 0.296402 & 0.078974 & 4.154304 & 0.046139 & 1.839942 \\
& ABE & 1.450809 & 0.057933 & 3.247697 & 0.062595 & 2.732703 \\
\hline & ALS & - & 2.654283 & 208.844190 & 2.540205 & 37.404072 \\
& ABT & - & 0.859905 & 40.190289 & 2.624051 & 97.059373 \\
$\mathbf{p = 2 0}$ & AHG & - & 0.101641 & 6.990070 & 0.114132 & 1.531057 \\
& ATE & - & 0.051824 & 2.590322 & 0.030506 & 0.655504 \\
& ABE & - & 0.032847 & 1.807182 & 0.036726 & 0.814317 \\
\hline & ALS & - & 1.744072 & 174.713509 & 1.779992 & 14.595444 \\
& ABT & - & 0.080129 & 6.798724 & 0.233602 & 1.666544 \\
$\mathbf{p = 1 0}$ & AHG & - & 0.051519 & 5.017843 & 0.072447 & 0.544649 \\
& ATE & - & 0.016758 & 1.216712 & 0.009415 & 0.076183 \\
& ABE & - & 0.011703 & 0.977689 & 0.011583 & 0.102156
\end{tabular}

Table 2: Standard deviation $\sigma=1.38$, error in comparison to simulation of size $n=10^{7}$. 


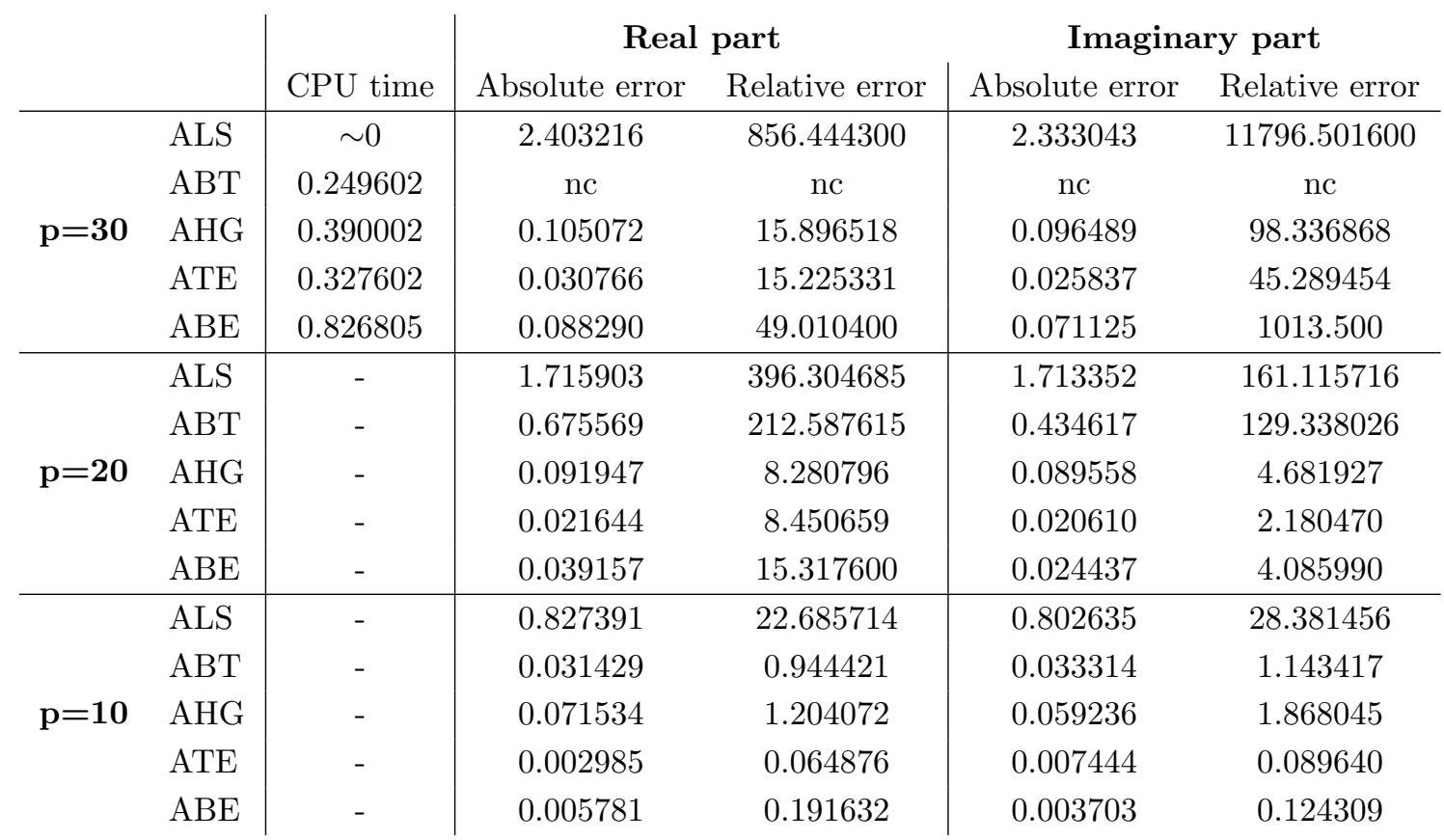

Table 3: Standard deviation $\sigma=0.70$, error in comparison to simulation of size $n=10^{7}$.

For $\sigma=2.3$, we have omitted the ALS method, since for standard deviation values of that size no sensible output was produced, as predicted by the original investigator [8. Similarly, we note that the truncated lognormal method becomes unstable for either too large $\sigma$ or $\omega$ values. Even though the ABT method has shown good performance for small parameter values with only moderate computational effort, the instability severely limits its operating range.

\begin{tabular}{ll|c|cc|cc} 
& & & \multicolumn{2}{|c}{ Real part } & \multicolumn{2}{c}{ Imaginary part } \\
& & CPU time & Absolute error & Relative error & Absolute error & Relative error \\
\hline & ALS & $\sim 0$ & 0.215030 & 466.752643 & 0.218099 & 1209.040113 \\
$\mathbf{p = 3 0}$ & ABT & 0.202801 & nc & nc & nc & nc \\
& AHG & 0.390002 & 0.119693 & 80.59962 & 0.118690 & 72.49969 \\
& ATE & 0.374402 & 0.050140 & 20.30160 & 0.058299 & 16.72387 \\
& ABE & 0.436803 & 0.124511 & 1785.250 & 0.072307 & 2572.940 \\
\hline $\mathbf{p = 2 0}$ & ALS & - & 0.182132 & 47.57666 & 0.175392 & 32.706530 \\
& ABT & - & 0.041104 & 28.65944 & 0.029539 & 25.00917 \\
& AHG & - & 0.114796 & 19.25712 & 0.113521 & 16.52755 \\
& ATE & - & 0.048757 & 2.92060 & 0.057162 & 2.341563 \\
ABE & - & 0.018411 & 11.41160 & 0.006797 & 4.51306 \\
\hline $\mathbf{p = 1 0}$ & ALS & - & 0.091365 & 2.103879 & 0.091022 & 1.652426 \\
& ABT & - & 0.002104 & 0.046956 & 0.001840 & 0.049079 \\
ATE & - & 0.084618 & 2.253233 & 0.076022 & 2.313334 \\
ABE & - & 0.047165 & 2.114443 & 0.054698 & 1.029962 \\
& & - & 0.002095 & 0.044906 & 0.001523 & 0.041799
\end{tabular}

Table 4: Standard deviation $\sigma=0.3$, error in comparison to simulation of size $n=10^{7}$.

The hypergeometric function formula was chosen as our benchmark method, as it performs well for any given parameter combination with comparatively small computational effort. The number of sum terms has been chosen in accordance with the specifications made by Beaulieu for their respective $\sigma$ values [8].

In contrast we can see the Bessel expansion approach being rather accurate for small values of $\omega$, while becoming more inaccurate for large values (especially for a small standard deviation). Nevertheless, we did manage to obtain good accuracy near $\omega=0$ or for large variances with this approach. 


\begin{tabular}{rl|c|cc|cc} 
& & & \multicolumn{2}{|c}{ Real part } & \multicolumn{2}{c}{ Imaginary part } \\
& & CPU time & Absolute error & Relative error & Absolute error & Relative error \\
\hline & ALS & 0.015600 & 0.007522 & 0.074963 & 0.006494 & 0.156856 \\
$\mathbf{p = 3 0}$ & ABT & 0.187201 & 0.003040 & 0.053925 & 0.006083 & 0.182432 \\
& AHG & 0.343202 & 0.099302 & 0.489928 & 0.101704 & 0.390938 \\
& ATE & 0.312002 & 0.013115 & 0.094753 & 0.011093 & 0.359235 \\
& ABE & 0.904806 & 0.307162 & 4.126880 & 0.232750 & 1.087630 \\
\hline & ALS & - & 0.004698 & 0.053300 & 0.003521 & 0.008572 \\
$\mathbf{p = 2 0}$ & ABT & - & 0.002020 & 0.046583 & 0.001494 & 0.004253 \\
& AHG & - & 0.021894 & 0.123830 & 0.020197 & 0.037871 \\
& ATE & - & 0.005960 & 0.055378 & 0.005177 & 0.012603 \\
& ABE & - & 0.001967 & 0.046330 & 0.001465 & 0.004213 \\
\hline & ALS & - & 0.000738 & 0.001572 & 0.000725 & 0.001679 \\
& ABT & - & 0.000187 & 0.000708 & 0.000242 & 0.000875 \\
AHG & - & 0.001397 & 0.003048 & 0.000953 & 0.002405 \\
& ATE & - & 0.001030 & 0.002032 & 0.000812 & 0.001916 \\
& ABE & - & 0.000187 & 0.000708 & 0.000242 & 0.000875
\end{tabular}

Table 5: Standard deviation $\sigma=0.05$, error in comparison to simulation of size $n=10^{7}$.

The vulnerability of the ABE approach to large $\omega$ values is most likely due to the Bessel function expansion diverging the further we stray from the origin. The Taylor approach does not appear to have this issue, since the expansion only needs to be locally stable in each individual interval $\left[a_{k}, a_{k+1}\right]$.

With adaptive interval and polynomial order settings (larger intervals for higher order polynomials, high order polynomials for high integrand oscillation), we managed to outperform the AHG benchmark method for the entirety of the tested values with slightly smaller CPU strain.

For an especially small standard deviation (for example, $\sigma=0.05$ ) the ALS shows great accuracy, with almost no computational effort (note that Mathematica sets 0.0156 per default as smallest time increment, hence we get values oscillating between 0 and 0.0156). As mentioned in [8] the closed form is only applicable for $\sigma$ converging to zero. The partial Taylor expansion of the ALS method is developed around its evaluation point $\sigma=0$, therefore the expansion is increasingly unreliable for larger $\sigma$ values. Beaulieu and Saberali have narrowed down the range where the closed form remains accurate to $\sigma<0.3$. Therefore the only two stable approximations, regardless of the range of their input parameter $\omega$ and $\sigma$, are the AHG and ATE approaches.

\section{$3 \quad$ Integral transformation and quadrature methods}

\subsection{Chebyshev-type quadrature}

Gubner [1] proposed a quadrature approach for the CF integration, which focused around the transformation of the integrand to a function which can be easily evaluated by numerical methods. The choice of a suitable integration method depends on the image of the transformation. In Table ?? we find a selection of commonly used quadrature formulas, as taken from Abramowitz and Stegun [1]. 


\begin{tabular}{c|c|c|c} 
Name & Interval & Orthogonal polynomials & Weights \\
\hline Gauss-Legendre quadrature & {$[-1,1]$} & Legendre polynomials & 1 \\
Gauss-Hermite quadrature & $(-\infty, \infty)$ & Hermite polynomials & $e^{-x^{2}}$ \\
Chebyshev-Gauss quadrature & $(-1,1)$ & Chebyshev polynomials (first kind) & $\frac{1}{\sqrt{1-x^{2}}}$ \\
Chebyshev-Gauss quadrature & {$[-1,1]$} & Chebyshev polynomials (second kind) & $\sqrt{1-x^{2}}$
\end{tabular}

Table 6: Common quadrature methods.

Gubner considers the $\mathrm{CF}$ as a contour integral, along the path $C=\{z(t)=t+i \pi / 2:-\infty<t<\infty\}$. From this derives ??:

$$
\begin{aligned}
\Phi_{Z}(\omega)=\int_{C} \frac{1}{\sqrt{2 \pi} \sigma z} e^{-\log (z)^{2} /(2 \sigma)^{2}} e^{i \omega z} d z & =\frac{\exp \left[(\pi /(2 \sigma))^{2} / 2\right]}{\sqrt{2 \pi} \sigma} \int_{\mathbb{R}} e^{-\omega e^{t}} e^{-i \omega t /(2 \sigma)^{2}} e^{-(t / \sigma)^{2}} d t \\
& =\frac{\exp \left[(\pi /(2 \sigma))^{2} / 2\right]}{\sqrt{\pi}} \underbrace{\int_{\mathbb{R}} e^{-\omega e^{\sqrt{2} \sigma x}} e^{-i \omega x /(2 \sigma)^{2}} e^{-x^{2}} d x}_{\psi(\omega)} .
\end{aligned}
$$

This formulation lends itself well to the Hermite-Gauss quadrature, through which we generate an approximation for $\psi(\omega)$. The weights $w_{k}$ for the approximation order $n$ can iteratively be generated by introducing the $k$ th root of the $n$th order Hermite polynomial $x_{k}^{(n)}$ into the $(n-1)$ th order polynomial:

$\psi(\omega) \approx \sum_{k=1}^{n} w_{k} e^{-\omega e^{\sqrt{2} \sigma x_{k}^{(n)}}} e^{-i \omega x_{k}^{(n)} /(2 \sigma)^{2}}$.

The original paper [11] suggests that the maximum accuracy is reached around an order of Hermite polynomial of $n=45$. The results of the quadrature become stationary beyond that point, which we were able to confirm in our replications.

Although the quadrature method showed great stability and fast evaluation times we will try improving upon this approach by introducing a slightly different transformation, and consequently quadrature method. We start out with ?? and introduce the remapping $x=L \cot (\theta)$, where $L$ is an approximation parameter [9]:

$$
\begin{aligned}
\psi(\omega)=\int_{\mathbb{R}} e^{-\omega e^{\sqrt{2} \sigma x}} e^{-i \omega x /(2 \sigma)^{2}} e^{-x^{2}} d x & =L \int_{0}^{\pi} \frac{1}{\sin ^{2}(\theta)} \underbrace{e^{-\omega e^{\sqrt{2} \sigma(L \cot \theta)}} e^{-i \omega L \cot (\theta) /(2 \sigma)^{2}} e^{-(L \cot (\theta))^{2}}}_{=g(L \cot \theta)} d \theta \\
& \approx \frac{L \pi}{N} \sum_{k=1}^{N-1} \frac{1}{\sin ^{2}(k \pi / N)} g\left(L \cot \left(\frac{k \pi}{N}\right)\right) .
\end{aligned}
$$

This approximation is essentially a specialisation of the Chebyshev-Gauss quadrature, which utilises the periodicity of the transformed integrand. The numerical integration may reach up to an exponential rate of convergence. The choice of $L$ can heavily influence the outcome of the approximation and the necessary number of terms. Through experimentation we have found $L \sim N$ or lower to be optimal for our purposes, achieving convergence in as little as 15 terms.

\subsection{Numerical results for quadrature methods}

We generate benchmark results as before in Section ?? and investigate the performance of the various quadrature and numerical integration approaches. 


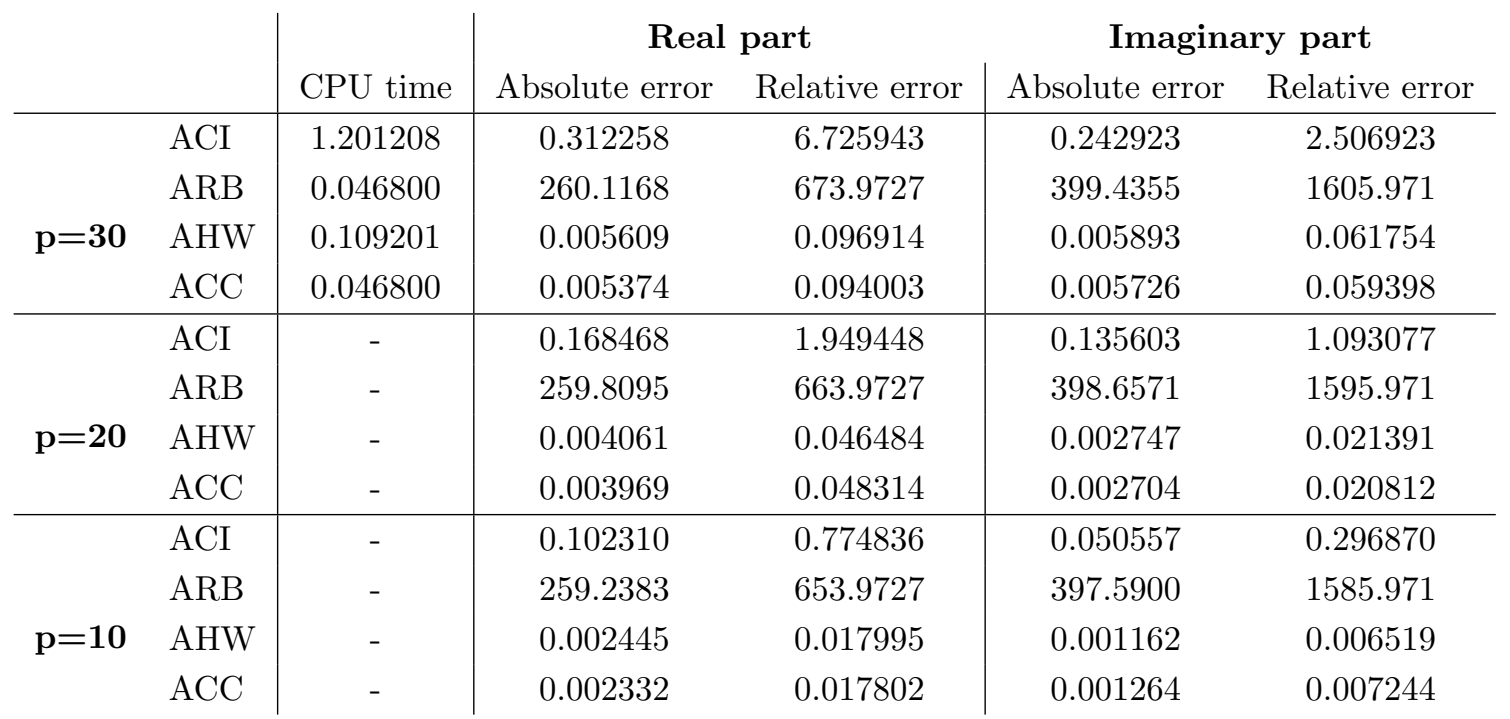

Table 7: Standard deviation $\sigma=2.30$, error in comparison to simulation of size $n=10^{7}$.

The method denoted by ACI was proposed by Beaulieu in "A simple integral form of lognormal characteristic functions convenient numerical computation" 4. The proposed reformulation of the CF was specifically constructed for easier numerical evaluation by the trapezoidal method. By ARB we refer to Barakat's approach utilizing Taylor and Hermite polynomials $[\underline{3}$.

\begin{tabular}{ll|c|cc|cc} 
& & & \multicolumn{2}{|c}{ Real part } & \multicolumn{2}{c}{ Imaginary part } \\
& & CPU time & Absolute error & Relative error & Absolute error & Relative error \\
\hline & ACI & 1.263608 & 0.030297 & 1.539971 & 0.056413 & 2.675018 \\
& ARB & 0.078001 & 74.46770 & 612.7762 & 54.14810 & 199.7956 \\
$\mathbf{p = 3 0}$ & AHW & 0.046800 & 0.005884 & 0.280866 & 0.005856 & 0.209535 \\
& ACC & $\sim 0$ & 0.005736 & 0.292645 & 0.005849 & 0.207896 \\
\hline & ACI & - & 0.017747 & 0.803512 & 0.029743 & 0.630684 \\
$\mathbf{p = 2 0}$ & ARB & - & 74.29133 & 602.7767 & 54.00300 & 189.7956 \\
& AHW & - & 0.004543 & 0.200430 & 0.003795 & 0.072062 \\
& ACC & - & 0.004549 & 0.220980 & 0.003820 & 0.072902 \\
\hline & ACI & - & 0.007221 & 0.381207 & 0.005600 & 0.044814 \\
$\mathbf{p = 1 0}$ & ARB & - & 74.03240 & 592.7762 & 53.5993 & 179.7956 \\
& AHW & - & 0.002234 & 0.112679 & 0.001735 & 0.013930
\end{tabular}

Table 8: Standard deviation $\sigma=1.38$, error in comparison to simulation of size $n=10^{7}$.

The AHW and ACC are both quadrature methods, one using Hermite-Gauss quadrature as stated by Gubner [11, whereas we devised the later approach based on a Chebyshev quadrature.

As we can see in the performance tables of higher valued standard deviation, the Barakat approach does not remain stable for arbitrary $\sigma$ values, despite producing good results for values of roughly $\sigma<0.5$ with little computational effort. 


\begin{tabular}{ll|c|cc|cc} 
& & & \multicolumn{2}{|c}{ Real part } & \multicolumn{2}{c}{ Imaginary part } \\
& & CPU time & Absolute error & Relative error & Absolute error & Relative error \\
\hline & ACI & 1.560010 & 0.006288 & 2.588322 & 0.004892 & 47.773835 \\
& ARB & 0.046800 & 1.568322 & 33.5406 & 3.459413 & 108.6734 \\
$\mathbf{p = 3 0}$ & AHW & 0.046800 & 0.005940 & 2.470303 & 0.004889 & 53.0809 \\
& ACC & 0.015600 & 0.005788 & 2.395781 & 0.004978 & 51.7819 \\
\hline & ACI & - & 0.004467 & 1.322967 & 0.002347 & 0.233787 \\
& ARB & - & 1.551014 & 23.5406 & 3.453067 & 98.6734 \\
$\mathbf{p = 2 0}$ & AHW & - & 0.004156 & 1.218673 & 0.002345 & 0.234062 \\
& ACC & - & 0.004009 & 1.153123 & 0.002383 & 0.241749 \\
\hline & ACI & - & 0.002176 & 0.039155 & 0.001227 & 0.041273 \\
& ARB & - & 1.518430 & 13.5387 & 3.346234 & 88.6735 \\
$\mathbf{p = 1 0}$ & AHW & - & 0.002178 & 0.039288 & 0.001234 & 0.041471 \\
& ACC & - & 0.002121 & 0.038010 & 0.001249 & 0.043617
\end{tabular}

Table 9: Standard deviation $\sigma=0.70$, error in comparison to simulation of size $n=10^{7}$.

The AHW approach serves as a benchmark quadrature method, and is able to produce accurate results in a runtime of less than 0.11 seconds for the 30 individual $\omega$ values, but failed to converge for $\sigma$ close to zero. Similarly, the ACC method struggles in the same area of values, as the approximation works much in the same way as the Hermite quadrature does.

However, with the ACC approach we are able to achieve an accuracy comparable to the AHW method (in $\sigma$ regions where both methods converge), in only a quarter of AHW's computation time. We attribute this to the correct choice of the parameter $L$ and the fact that no weights or nodes have to be pre-determined for evaluation.

\begin{tabular}{ll|c|cc|cc} 
& & & \multicolumn{2}{|c}{ Real part } & \multicolumn{2}{c}{ Imaginary part } \\
& & CPU time & Absolute error & Relative error & Absolute error & Relative error \\
\hline & ACI & 1.201208 & 0.004813 & 18.372205 & 0.005173 & 16.355073 \\
& ARB & 0.046800 & 0.066545 & 18.875980 & 0.069413 & 17.206620 \\
$\mathbf{p = 1 0}$ & AHW & 0.046800 & 0.004813 & 18.371627 & 0.005172 & 16.344597 \\
& ACC & 0.015600 & 0.004845 & 17.998674 & 0.005131 & 16.337516 \\
\hline & ACI & - & 0.003436 & 0.862905 & 0.004030 & 1.359070 \\
& ARB & - & 0.064301 & 8.115747 & 0.066770 & 7.030268 \\
$\mathbf{p = 2 0}$ & AHW & - & 0.003436 & 0.862865 & 0.004030 & 1.359085 \\
& ACC & - & 0.003477 & 0.866225 & 0.003988 & 1.342209 \\
\hline & ACI & - & 0.002080 & 0.044183 & 0.001519 & 0.041719 \\
& ARB & - & 0.036754 & 1.434342 & 0.041446 & 1.011095 \\
$\mathbf{p = 1 0}$ & AHW & - & 0.002080 & 0.044184 & 0.001519 & 0.041719 \\
& ACC & - & 0.002122 & 0.044253 & 0.001548 & 0.045194
\end{tabular}

Table 10: Standard deviation $\sigma=0.30$, error in comparison to simulation of size $n=10^{7}$. 


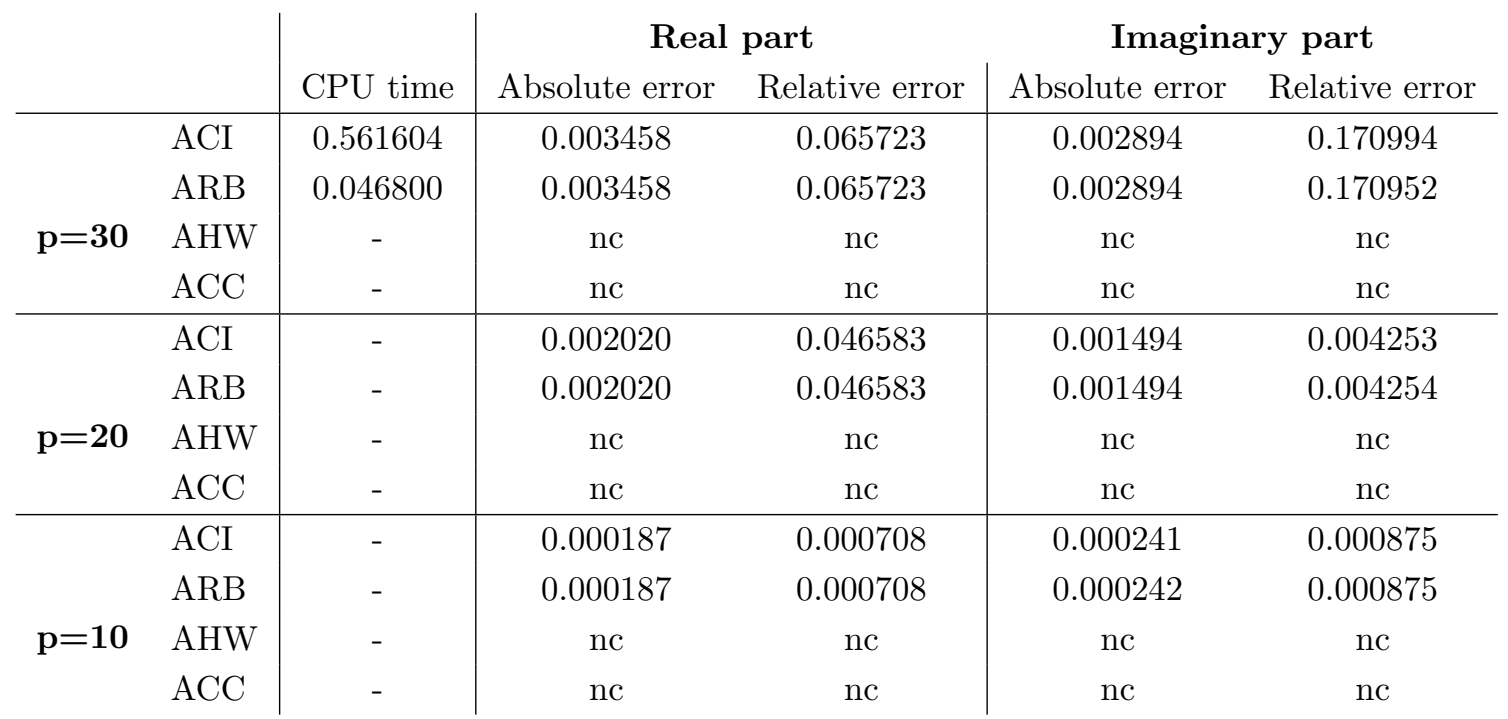

Table 11: Standard deviation $\sigma=0.05$, error in comparison to simulation of size $n=10^{7}$.

\section{Conclusions}

In Sections ?? and ??, we have introduced new approximation approaches and compared them to existing methods. While the Bessel function based approximation exhibited the greatest accuracy for specific parameter values, both the ATE and ACC approaches are more widely applicable due to their stability. With the exception of $\sigma$ values very close to zero $(<0.25)$, the Chebyshev quadrature has shown the best overall performance. We believe that pairing the Chebyshev quadrature for larger variances with the ALS approach for small variances may provide a strong technique for the accurate computation of the lognormal CF for arbitrary parameter values. Given that we may not know the parameters for which the chosen approximation must perform accurately, the Taylor expansion approach provides a viable alternative, taking into account its computational effort, accuracy and stability across the entirety of tested parameter ranges. While the combination method of quadrature and closed form approaches might offer a quick and accurate evaluation, the vulnerability of the local approximations to certain ranges of standard deviations may not offer enough stability for some applications.

We did consider Filon-type integration for highly oscillatory integrands with $\sigma<0.25$, suggested by Iserles and Norsett [13, but the accuracy and simplicity of computation inherent to the ALS method in this range makes it the preferred bridge for the gap the quadrature methods leave open.

The only approaches which are truly applicable to arbitrary domains of values are the partial Taylor expansion, the hypergeometric function approach and Beaulieu's convenient integral formulation, amongst which we have determined the Taylor expansion to be the most efficient way of computation.

\section{Acknowledgments}

The authors would like to thank the Editor and the two referees for careful reading and comments which improved the paper. We would like to express further thanks to Aaron Russell and Puneet Matharu of the University of Manchester for their careful reading of this paper.

\section{References}

[1] M. Abramowitz, I. Stegun, "Handbook of Mathematical Functions with Formulas, Graphs, and Mathematical Tables - Chapter 25.4, Integration", United States Department of Commerce, National Bureau of Standards (NBS), 1964.

[2] S. Asmussen, J. L. Jensen, L. Rojas-Nandayapa, "On the Laplace transform of the lognormal distribution", Methodology and Computing in Applied Probability, vol. 18, pp. 441-458, June 2016. 
[3] R. Barakat, "Sums of independent lognormally distributed random variables", Journal of the Optical Society of America, vol. 66, pp. 211-216, 1976.

[4] N. C. Beaulieu, "A simple integral form of lognormal characteristic functions convenient for numerical computation", in Proceedings of the IEEE GLOBECOM '06, San Francisco, CA, pp. 1-3, November 2006.

[5] N. C. Beaulieu, "Fast convenient numerical computation of lognormal characteristic functions", IEEE Transactions on Communications, vol. 56, pp. 331-333, March 2008.

[6] N. C. Beaulieu, "A power series expansion for the truncated lognormal characteristic function", 25th Biennial Symposium on Communications, May 2010.

[7] N. C. Beaulieu, "An extended limit theorem for correlated lognormal sums", IEEE Transactions on Communications, vol. 60, pp. 23-26, January 2012.

[8] N. C. Beaulieu, S. A. Saberali, "New approximations to the lognormal characteristic function", Globecom 2012 - Communication Theory Symposium, pp. 2168-2172.

[9] John P. Boyd, "Exponentially convergent Fourier-Chebshev quadrature schemes on bounded and infinite intervals", Journal of Scientific Computing, vol. 2, pp. 99-109, 1987.

[10] F. Clementi, M. Gallegati, "Pareto's law of income distribution: Evidence for Germany, the United Kingdom, and the United States", EconWPA, 2005.

[11] J. A. Gubner, "A new formula for lognormal characteristic functions", IEEE Transactions on Vehicular Technology, vol. 55, pp. 1668-1671, September 2006.

[12] F. Heliot, X. Chu, R. Hoshyar, R. Tafazolli, "A tight closed-form approximation of the log-normal fading channel capacity", IEEE Transaction on Wireless Communications, vol. 8, pp. 2842-2847, June 2009.

[13] A. Iserles, S. P. Norsett, "On quadrature methods for highly oscillatory functions and their implementation", BIT, vol. 40, pp. 1-4, 2000.

[14] E. Limpert, W. A. Stahel, M. Abbt, "Lognormal distributions across the sciences: Keys and clues", BioScience, vol. 51, pp. 341-352, May 2001.

[15] A. Papoulis, S. U. Pillai, "Probability, Random Variables and Stochastic Processes", New York: McGraw-Hill, 2002.

[16] T. S. Rappaport, "Wireless Communications - Principles and Practice", New Jersey: Prentice Hall, 1996.

[17] C. Tellambura, D. Senaratne, "Accurate computation of the MGF of the lognormal distribution and its application to sum of lognormals", IEEE Transactions on Communications, vol. 58, pp. 1568-1577, May 2010.

[18] Y. Yeh, S. C. Schwartz, "Outage probability in mobile telephony due to multiple lognormal interferers", IEEE Transactions on Communications, vol. 32, pp. 380-388, April 1984. 